\title{
A Simplification for Exp-Function Method When the Balanced Nonlinear Term Is a Certain Product
}

\author{
Hong-Zhun Liu \\ Zhijiang College, Zhejiang University of Technology, Hangzhou 310024, China \\ Correspondence should be addressed to Hong-Zhun Liu; mathlhz@163.com
}

Received 24 October 2013; Revised 8 December 2013; Accepted 8 December 2013

Academic Editor: Hossein Jafari

Copyright (C) 2013 Hong-Zhun Liu. This is an open access article distributed under the Creative Commons Attribution License, which permits unrestricted use, distribution, and reproduction in any medium, provided the original work is properly cited.

The Exp-function method plays an important role in searching for analytic solutions of many nonlinear differential equations. In this paper, we prove that the balancing procedure in the method is unnecessary when the balanced nonlinear term is a product of the dependent variable under consideration and its derivatives. And in this case, the ansatz of the method can be simplified to be with less parameters so as to be easy to calculate.

\section{Introduction}

In $2006, \mathrm{He}$ and $\mathrm{Wu}$ firstly proposed the so-called Expfunction method to search for solitary solutions and periodic solutions of nonlinear partial differential equations (PDE) [1]. This method soon drew the attention of many researchers and was successfully applied to many nonlinear problems [2-31]. Among them, it is worth mentioning that Zhu firstly applied this method to difference-differential equations, which shows that the method is also effective in this case $[8,9]$. After Zhu, Dai et al. generalized the Exp-function method to find new exact traveling wave solutions of nonlinear PDE and nonlinear differential-difference equations [19]. Recently, $\mathrm{Ma}$ et al. extended the Exp-function method to multiple Expfunction method for constructing multiple wave solutions $[32,33]$. He elucidated how to solve fractional differential equations with local fractional derivatives via the fractional complex transformation and the Exp-function method [34].

For convenience, we first introduce the Exp-function method in brief.

1.1. Outline of the Exp-Function Method. Suppose that we consider a (1+1)-dimensional nonlinear PDE in the form

$$
E\left(u, u_{t}, u_{x}, u_{x x}, u_{x t}, u_{t t}, \ldots\right)=0 .
$$

Using traveling wave transformation

$$
u=u(\eta), \quad \eta=k x+\omega t
$$

we get a nonlinear ordinary differential equation (ODE)

$$
P\left(u, u^{\prime}, u^{\prime \prime}, \ldots\right)=0 \text {, }
$$

where the prime, as it is in the following, denotes the derivation with respect to $\eta$.

The Exp-function method is based on the assumption that the solutions of (3) can be expressed in the following form:

$$
\begin{aligned}
u(\eta) & =\frac{\sum_{n=-c}^{d} a_{n} \exp (n \eta)}{\sum_{n=-p}^{q} b_{n} \exp (n \eta)} \\
& =\frac{a_{-c} \exp (-c \eta)+\cdots+a_{d} \exp (d \eta)}{b_{-p} \exp (-p \eta)+\cdots+b_{q} \exp (q \eta)},
\end{aligned}
$$

where $c, d, p$, and $q$ are positive integers to be determined and $a_{n}$ and $b_{n}$ are constants to be specified.

Then we can express the highest order nonlinear and linear terms in (3) in terms of (4). In the resulting terms, determine $d$ and $q$ through balancing the highest order Expfunction and $c$ and $p$ by balancing the lowest order one.

Substituting (4) along with the determined $c, d$, p, and $q$ into (3), we can obtain an equation for $\exp (\eta)$. Setting all the coefficients of the different powers of $\exp (\eta)$ to zero leads to a set of algebraic equations for $a_{n}, b_{n}, k$, and $\omega$. Determine values of $a_{n}, b_{n}, k$, and $\omega$ by solving this algebraic equations and put these values into (4). Thus we may obtain nontrivial exact traveling wave solutions of (1). 
1.2. An Open Problem. Among the Exp-function method, the balancing computation is laborious but prior. However, we observe that the balancing procedure of the Exp-function method in studied examples always leads to the same case $c=p$ and $d=q$ [21]. This fact has partly been proved in $[16,22]$. In [16], Ali has proved it by assuming the highest order linear and nonlinear terms as $u^{(n)}$ and $u^{r} u^{(s)}(s<n)$, respectively. In [22], making use of the same approach as was done by Ebaid proved the fact for nonlinear terms in the form $u^{\gamma}(\gamma \geqslant 2), u^{(s)} u^{k}(s, k \geqslant 1),\left[u^{(s)}\right]^{\Omega}(s \geqslant 1, \Omega \geqslant 2)$, and $\left[u^{(s)}\right]^{\Omega} u^{\lambda}(s, \Omega, \lambda \geqslant 1)$, respectively, along with linear term $u^{(r)}(r \geqslant 1)$. Ebaid claimed in the abstract and section "Conclusions" of his article that the case $c=p$ and $d=q$ is the only relation that "can be obtained through applying the Exp-function ansatz for all possible cases of nonlinear ODEs."

"However, one cannot construct a general form for the highest order nonlinear term because there are many possibilities other than the ones considered" [21]; Aslan and Marinakis concluded that these authors just took some special cases of the nonlinear term into account and hence the problem still remained open. In this paper, we will construct a special case to show that Ebaid's claim is not true; namely, the case $c=p$ and $d=q$ is not the only relation for some special differential equations and hence the problem is still open.

In what follows, we will discuss the relations of $c, d, p$, and $q$ in a more effective and concise way.

\section{Main Result}

2.1. Some Terminology. To begin with, we recall some terminology in [35].

A monomial in a collection of variables $x_{1}, \ldots, x_{n}$ is a product

$$
x_{1}^{\alpha_{1}} x_{2}^{\alpha_{2}} \ldots x_{n}^{\alpha_{n}}
$$

where the $\alpha_{i}$ are nonnegative integers.

The total degree of (5) is the sum of the exponents: $\alpha_{1}+$ $\cdots+\alpha_{n}$.

A polynomial is said to be homogeneous if all the monomials appearing in it with nonzero coefficients have the same total degree.

For instance, $u^{2} u^{\prime} u^{\prime \prime 3}$ is a product of $u$ and its derivatives $u^{\prime}$ and $u^{\prime \prime}$, and its total degree is $6 . u^{2}+u^{\prime 2}+u u^{\prime \prime}$ is homogeneous.

2.2. Two Introducing Ansatz Function. For convenience, we assume $v(\eta)$ is expressed in the form

$$
\begin{aligned}
v(\eta) & =\frac{\sum_{n=-c_{1}}^{d_{1}} k_{n} \exp (n \eta)}{\sum_{n=-p_{1}}^{q_{1}} l_{n} \exp (n \eta)} \\
& =\frac{k_{-c_{1}} \exp \left(-c_{1} \eta\right)+\cdots+k_{d_{1}} \exp \left(d_{1} \eta\right)}{l_{-p_{1}} \exp \left(-p_{1} \eta\right)+\cdots+l_{q_{1}} \exp \left(q_{1} \eta\right)},
\end{aligned}
$$

where $c_{1}, d_{1}, p_{1}$, and $q_{1}$ are positive integers to be determined and $k_{n}$ and $l_{n}$ are constants to be specified.
The following three formulas will be used in this section:

$$
\begin{aligned}
& u^{\prime}(\eta) \\
& =\frac{(p-c) a_{-c} b_{-p} \exp (-(c+p) \eta)+\cdots+(d-q) a_{d} b_{q} \exp ((d+q) \eta)}{b_{-p}^{2} \exp (-2 p \eta)+\cdots+b_{q}^{2} \exp (2 q \eta)},
\end{aligned}
$$

$$
\begin{aligned}
& u(\eta) \cdot v(\eta) \\
& =\frac{a_{-c} k_{-c_{1}} \exp \left(-\left(c+c_{1}\right) \eta\right)+\cdots+a_{d} k_{d_{1}} \exp \left(\left(d+d_{1}\right) \eta\right)}{b_{-p} l_{-p_{1}} \exp \left(-\left(p+p_{1}\right) \eta\right)+\cdots+b_{q} l_{q_{1}} \exp \left(\left(q+q_{1}\right) \eta\right)}
\end{aligned}
$$

$$
\begin{aligned}
& \frac{u(\eta)}{v(\eta)} \\
& =\frac{a_{-c} l_{-p_{1}} \exp \left(-\left(c+p_{1}\right) \eta\right)+\cdots+a_{d} l_{q_{1}} \exp \left(\left(d+q_{1}\right) \eta\right)}{b_{-p} k_{-c_{1}} \exp \left(-\left(p+c_{1}\right) \eta\right)+\cdots+b_{q} k_{d_{1}} \exp \left(\left(q+d_{1}\right) \eta\right)}
\end{aligned}
$$

Before presenting our definitions, we recall an important fact that the constants $a_{-c}, a_{d}, b_{-p}$, and $b_{q}$ in the ansatz of (4) can be assumed to be nonzero during the process of balancing the linear term of highest order with the highest order nonlinear term of certain ODE, and so do the constants $k_{-c_{1}}, k_{d}, l_{-p_{1}}$, and $l_{q}$ in ansatz (6). Hence, in this paper, we assume that all above eight constants are nonzero.

Therefore, for ansatz (4), we can define the ansatz functions $L(\cdot)$ and $R(\cdot)$ as follows:

$$
\begin{gathered}
L(u)=L\left(\frac{a_{-c} \exp (-c \eta)+\cdots+a_{d} \exp (d \eta)}{b_{-p} \exp (-p \eta)+\cdots+b_{q} \exp (q \eta)}\right) \\
=-c-(-p)=p-c, \\
R(u)=R\left(\frac{a_{-c} \exp (-c \eta)+\cdots+a_{d} \exp (d \eta)}{b_{-p} \exp (-p \eta)+\cdots+b_{q} \exp (q \eta)}\right)=d-q .
\end{gathered}
$$

In particular, we define $L(C)=R(C)=0$ for arbitrary constant $C$.

For example, we have

$$
\begin{aligned}
& L\left(\frac{\exp (-\eta)+\exp (2 \eta)}{\exp (-2 \eta)+\exp (2 \eta)}\right)=1, \\
& R\left(\frac{\exp (-\eta)+\exp (2 \eta)}{\exp (-2 \eta)+\exp (2 \eta)}\right)=0 .
\end{aligned}
$$

According to the definitions, we can find that in ansatz (4) $c=$ $p$ equals $L(u)=0$ and $d=q$ equals $R(u)=0$. Therefore, the open problem for ansatz (4) is equal to whether the relations $L(u)=0$ and $L(u)=0$ hold. 
2.3. Properties of the Ansatz Functions. Assuming $c \neq p, d \neq q$, $c_{1} \neq p_{1}$, and $d_{1} \neq q_{1}$ in ansatz (4) and (6), from (7), we obtain

$$
\begin{aligned}
& L\left(u^{\prime}\right)=2 p-(c+p)=p-c=L(u), \\
& R\left(u^{\prime}\right)=(d+q)-2 q=d-q=R(u) .
\end{aligned}
$$

So we have

$$
\begin{aligned}
& L(u)=L\left(u^{\prime}\right)=L\left(u^{\prime \prime}\right)=\cdots=L\left(u^{(n)}\right), \\
& R(u)=R\left(u^{\prime}\right)=\cdots=R\left(u^{(n)}\right),
\end{aligned}
$$

for arbitrary nonnegative integer $n$.

And from (8) and (9), we have

$$
\begin{aligned}
L(u \cdot v) & =\left(p+p_{1}\right)-\left(c+c_{1}\right) \\
& =(p-c)+\left(p_{1}-c_{1}\right) \\
& =L(u)+L(v), \\
R(u \cdot v) & =\left(d+d_{1}\right)-\left(q+q_{1}\right) \\
& =(d-q)+\left(d_{1}-q_{1}\right) \\
& =R(u)+R(v), \\
L\left(\frac{u}{v}\right) & =\left(p+c_{1}\right)-\left(c+p_{1}\right) \\
& =(p-c)-\left(p_{1}-c_{1}\right) \\
& =L(u)-L(v), \\
R\left(\frac{u}{v}\right) & =\left(d+q_{1}\right)-\left(q+d_{1}\right) \\
& =(d-q)-\left(d_{1}-q_{1}\right) \\
& =R(u)-R(v) .
\end{aligned}
$$

Hence, we have

$$
L\left(u^{\kappa}\right)=\kappa \cdot L(u), \quad R\left(u^{\kappa}\right)=\kappa \cdot R(u),
$$

for any integer $\kappa$.

2.4. Theorem and Proof. In this section, we assume the balanced nonlinear term is a product of dependent variable $u$ and its derivatives; namely,

$$
u^{i_{1}}\left(u^{\prime}\right)^{i_{2}} \cdots\left(u^{(m)}\right)^{i_{m}}
$$

where $i_{j}(j=1, \ldots, m)$ are nonnegative integers. The fact that the product (16) is a nonlinear term implies $i_{1}+i_{2}+\cdots+i_{m} \geqslant 2$. In other words, the total degree of (16) is at least 2.

Theorem 1. Suppose that the balanced nonlinear term in (3) is a product of $u$ and its derivatives in the form of (16) and the balancing linear term is $u^{(s)}$, where $s$ is a nonnegative integer; then the Exp-function ansatz (4) admits $L(u)=0$ and $R(u)=$ 0 .
Proof. By contradiction, suppose that $L(u) \neq 0$. Then we have

$$
\begin{aligned}
& L\left(u^{i_{1}}\left(u^{\prime}\right)^{i_{2}} \cdots\left(u^{(m)}\right)^{i_{m}}\right) \\
& =L\left(u^{i_{1}}\right)+L\left(\left(u^{\prime}\right)^{i_{2}}\right)+\cdots+L\left(\left(u^{(m)}\right)^{i_{m}}\right) \\
& =i_{1} \cdot L(u)+i_{2} \cdot L\left(u^{\prime}\right)+\cdots+i_{m} \cdot L\left(u^{(m)}\right) \\
& =i_{1} \cdot L(u)+i_{2} \cdot L(u)+\cdots+i_{m} \cdot L(u) \\
& =\left(i_{1}+i_{2}+\cdots+i_{m}\right) \cdot L(u), \\
& L\left(u^{(s)}\right)=L(u) .
\end{aligned}
$$

Balancing linear and nonlinear terms requires

$$
L\left(u^{i_{1}}\left(u^{\prime}\right)^{i_{2}} \ldots\left(u^{(m)}\right)^{i_{m}}\right)=L\left(u^{(s)}\right) .
$$

So we obtain

$$
\left(i_{1}+i_{2}+\cdots+i_{m}-1\right) \cdot L(u)=0 .
$$

Since $i_{1}+i_{2}+\cdots+i_{m}-1 \neq 0$, we arrive at the result $L(u)=0$. This is a contradiction.

The result $R(u)=0$ can be obtained in a similar way; here we omit the details.

Remark. Since our forms of the linear and nonlinear terms are in a more general setting, Theorem 1 covers the results presented by Ali and Ebaid, respectively.

2.5. A Simplification for the Exp-Function Method. According to Theorem 1, if the balanced nonlinear term is a product, ansatz (4) can be reduced to the following equivalent form:

$$
u=\frac{\alpha_{0}+\alpha_{1} \exp (\eta)+\cdots+\alpha_{\tau} \exp (\tau \eta)}{1+\beta_{1} \exp (\eta)+\cdots+\beta_{\tau} \exp (\tau \eta)},
$$

where $\tau$ is a free positive integer with $\tau \geqslant 2$ and $\alpha_{i}$ and $\beta_{i}$ are constants to be specified. Ansatz (20) is concise and easy to calculate and makes the Exp-function method more straightforward.

For example, Naher et al. applied the Exp-function method to constructing the traveling wave solutions of the $(3+1)$-dimensional modified KdV-Zakharov-Kuznetsev equation in the form [25]

$$
u_{t}+\alpha u^{2} u_{x}+u_{x x x}+u_{x y y}+u_{x z z}=0 .
$$

The traveling wave transformation

$$
u(x, y, z, t)=u(\eta), \quad \eta=x+y+z-V t
$$

carries (21) into an ODE

$$
-V u^{\prime}+\alpha u^{2} u^{\prime}+3 u^{\prime \prime \prime}=0 .
$$

Since the nonlinear term $u^{2} u^{\prime}$ is a product of $u$ and $u^{\prime}$, we can immediately assume ansatz (4) in the form

$$
u=\frac{\alpha_{0}+\alpha_{1} \exp (\eta)+\alpha_{2} \exp (2 \eta)}{1+\beta_{1} \exp (\eta)+\beta_{2} \exp (2 \eta)}
$$

which is equivalent to the case $c=p=1$ and $d=q=1$ and in accord with Naher's (namely, (3.8) in [25]). 


\section{A Counter-Example}

In [22], Ebaid claimed in his abstract that the case $c=p$ and $d=q$ was the only relation that could be obtained by applying this method to any nonlinear ODE. In this section, we construct a counter-example to show that the case $c=p$ and $d=q$ is not the only relation. In fact, the claim does not applied to each nonlinear homogeneous ODE.

For example, we can create the following ODE:

$$
u^{2}+u^{\prime 2}+u u^{\prime \prime}=0
$$

which can be rewritten as

$$
u+\frac{u^{\prime 2}}{u}+u^{\prime \prime}=0 .
$$

We have

$$
\begin{array}{ll}
L\left(\frac{u^{\prime 2}}{u}\right)=L(u), & L\left(u^{\prime \prime}\right)=L(u), \\
R\left(\frac{u^{\prime 2}}{u}\right)=R(u), & R\left(u^{\prime \prime}\right)=R(u) .
\end{array}
$$

From (27), the relations $L\left(u^{\prime 2} / u\right)=L\left(u^{\prime \prime}\right)$ and $R\left(u^{\prime 2} / u\right)=$ $R\left(u^{\prime \prime}\right)$ hold automatically. Hence by balancing nonlinear term $u^{\prime 2} / u$ and linear term $u^{\prime \prime}$, we cannot determine the relations of $c, d, p$, and $q$. That is to say, all of them are free constants. Hence it is possible that the relation $c \neq p$ and $d \neq q$ holds. In other words, the relation $c=p$ and $d=q$ is not all-inclusive.

\section{Conclusion}

In summary, we present an entire novel approach to prove that the balancing procedure in Exp-function method is unnecessary when the balanced nonlinear term is a product of the dependent variable under consideration and its derivatives. Our results cover the results presented by Ali and Ebaid. We believe that our work can serve as an answer to the open problem proposed by Aslan et al.

\section{Acknowledgments}

Many thanks are due to the helpful comments and suggestions from the anonymous referees and support from the NSF of China (11201427) and the College Foundation of Zhijiang College of Zhejiang University of Technology (YJJ0819).

\section{References}

[1] J.-H. He and X.-H. Wu, "Exp-function method for nonlinear wave equations," Chaos, Solitons \& Fractals, vol. 30, no. 3, pp. 700-708, 2006.

[2] S. Zhang, "Application of Exp-function method to a KdV equation with variable coefficients," Physics Letters A, vol. 365, no. 5-6, pp. 448-453, 2007.

[3] J.-H. He and M. A. Abdou, "New periodic solutions for nonlinear evolution equations using Exp-function method," Chaos, Solitons \& Fractals, vol. 34, no. 5, pp. 1421-1429, 2007.
[4] A. Bekir and A. Boz, "Exact solutions for a class of nonlinear partial differential equations using exp-function method," International Journal of Nonlinear Sciences and Numerical Simulation, vol. 8, no. 4, pp. 505-512, 2007.

[5] M. A. Abdou, A. A. Soliman, and S. T. El-Basyony, "New application of Exp-function method for improved Boussinesq equation," Physics Letters A, vol. 369, no. 5-6, pp. 469-475, 2007.

[6] A. Ebaid, "Exact solitary wave solutions for some nonlinear evolution equations via Exp-function method," Physics Letters A, vol. 365, no. 3, pp. 213-219, 2007.

[7] S. A. El-Wakil, M. A. Madkour, and M. A. Abdou, "Application of Exp-function method for nonlinear evolution equations with variable coefficients," Physics Letters A, vol. 369, no. 1-2, pp. 62 69, 2007.

[8] S.-D. Zhu, "Exp-function method for the Hybrid-Lattice system," International Journal of Nonlinear Sciences and Numerical Simulation, vol. 8, no. 3, pp. 461-464, 2007.

[9] S.-D. Zhu, "Exp-function method for the discrete mKdV lattice," International Journal of Nonlinear Sciences and Numerical Simulation, vol. 8, no. 3, pp. 465-468, 2007.

[10] X.-H. Wu and J.-H. He, "Solitary solutions, periodic solutions and compacton-like solutions using the Exp-function method," Computers \& Mathematics with Applications, vol. 54, no. 7-8, pp. 966-986, 2007.

[11] X.-H. Wu and J.-H. He, "EXP-function method and its application to nonlinear equations," Chaos, Solitons \& Fractals, vol. 38, no. 3, pp. 903-910, 2008.

[12] A. Bekir and A. Boz, "Exact solutions for nonlinear evolution equations using Exp-function method," Physics Letters A, vol. 372, no. 10, pp. 1619-1625, 2008.

[13] J.-H. He, "An elementary introduction to recently developed asymptotic methods and nanomechanics in textile engineering," International Journal of Modern Physics B, vol. 22, no. 21, pp. 3487-3578, 2008.

[14] F. Xu, "Application of Exp-function method to symmetric regularized long wave (SRLW) equation," Physics Letters A, vol. 372, no. 3, pp. 252-257, 2008.

[15] S. Zhang, "Application of Exp-function method to Riccati equation and new exact solutions with three arbitrary functions of Broer-Kaup-Kupershmidt equations," Physics Letters A, vol. 372, no. 11, pp. 1873-1880, 2008.

[16] A. T Ali, "A note on the Exp-function method and its application to nonlinear equations," Physica Scripta, vol. 79, no. 2, Article ID 025006, 2009.

[17] L. M. B. Assas, "New exact solutions for the Kawahara equation using Exp-function method," Journal of Computational and Applied Mathematics, vol. 233, no. 2, pp. 97-102, 2009.

[18] A. Bekir and A. Boz, "Application of Exp-function method for $(2+1)$-dimensional nonlinear evolution equations," Chaos, Solitons and Fractals, vol. 40, no. 1, pp. 458-465, 2009.

[19] C.-Q. Dai, X. Cen, and S.-S. Wu, "Exact travelling wave solutions of the discrete sine-Gordon equation obtained via the exp-function method," Nonlinear Analysis. Theory, Methods \& Applications A, vol. 70, no. 1, pp. 58-63, 2009.

[20] S. Zhang and H.-Q. Zhang, "Exp-function method for Nsoliton solutions of nonlinear differential-difference equations," Zeitschrift fur Naturforschung A, vol. 65, no. 11, pp. 924-934, 2010.

[21] I. Aslan and V. Marinakis, "Some remarks on exp-function method and its applications," Communications in Theoretical Physics, vol. 56, no. 3, pp. 397-403, 2011. 
[22] A. Ebaid, "An improvement on the Exp-function method when balancing the highest order linear and nonlinear terms," Journal of Mathematical Analysis and Applications, vol. 392, no. 1, pp. 15, 2012.

[23] J.-M. Kim and C. Chun, "New exact solutions to the KdVBurgers-Kuramoto equation with the Exp-function method," Abstract and Applied Analysis, vol. 2012, Article ID 892420, 10 pages, 2012.

[24] H. Jafari, N. Kadkhoda, and C. M. Khalique, "Exact solutions of $\phi^{4}$ equation using lie symmetry approach along with the simplest equation and exp-function methods," Abstract and Applied Analysis, vol. 2012, Article ID 350287, 7 pages, 2012.

[25] H. Naher, F. A. Abdullah, and M. A. Akbar, "New traveling wave solutions of the higher dimensional nonlinear partial differential equation by the exp-function method," Journal of Applied Mathematics, vol. 2012, Article ID 575387, 14 pages, 2012.

[26] L. Zhao, D. Huang, and S. Zhou, "A new algorithm for automatic computation of solitary wave solutions to nonlinear partial differential equations based on the Exp-function method," Applied Mathematics and Computation, vol. 219, no. 4, pp. 18901896, 2012.

[27] J.-H. He, "Asymptotic methods for solitary solutions and compactons," Abstract and Applied Analysis, vol. 2012, Article ID 916793, 130 pages, 2012.

[28] A. Bekir and E. Aksoy, "Exact solutions of extended shallow water wave equations by exp-function method," International Journal of Numerical Methods for Heat \& Fluid Flow, vol. 23, no. 2, pp. 305-319, 2013.

[29] A. H. Bhrawy, A. Biswas, M. Javidi, W. X. Ma, Z. Pınar, and A. Yildırım, "New solutions for $(1+1)$-dimensional and $(2+1)$-dimensional Kaup-Kupershmidt equations," Results in Mathematics, vol. 63, no. 1-2, pp. 675-686, 2013.

[30] K. Khan and M. Ali Akbar, "Traveling wave solutions of the (2+ 1)-dimensional Zoomeron equation and the Burgers equations via the MSE method and the Exp-function method," Ain Shams Engineering Journal. In press.

[31] G. Magalakwe and C. M. Khalique, "New exact solutions for a generalized double sinh-Gordon equation," Abstract and Applied Analysis, vol. 2013, Article ID 268902, 5 pages, 2013.

[32] W.-X. Ma, T. Huang, and Y. Zhang, "A multiple exp-function method for nonlinear differential equations and its application," Physica Scripta, vol. 82, no. 6, Article ID 065003, 2010.

[33] W.-X. Ma and Z. Zhu, "Solving the $(3+1)$-dimensional generalized KP and BKP equations by the multiple exp-function algorithm," Applied Mathematics and Computation, vol. 218, no. 24, pp. 11871-11879, 2012.

[34] J.-H. He, "Exp-function Method for Fractional Differential Equations," International Journal of Nonlinear Sciences and Numerical Simulation, vol. 14, no. 6, pp. 363-366, 2013.

[35] D. A. Cox, J. Little, and D. O'Shea, Using Algebraic Geometry, vol. 185, Springer, New York, NY, USA, 2nd edition, 2005. 


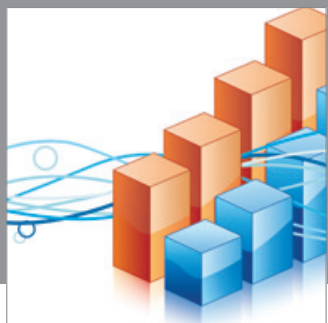

Advances in

Operations Research

mansans

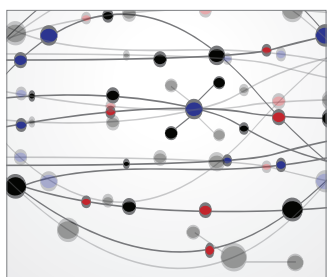

The Scientific World Journal
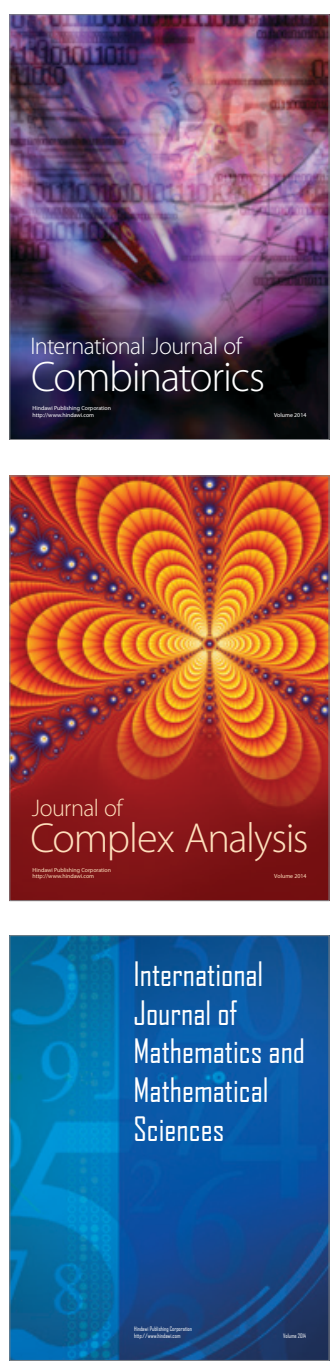
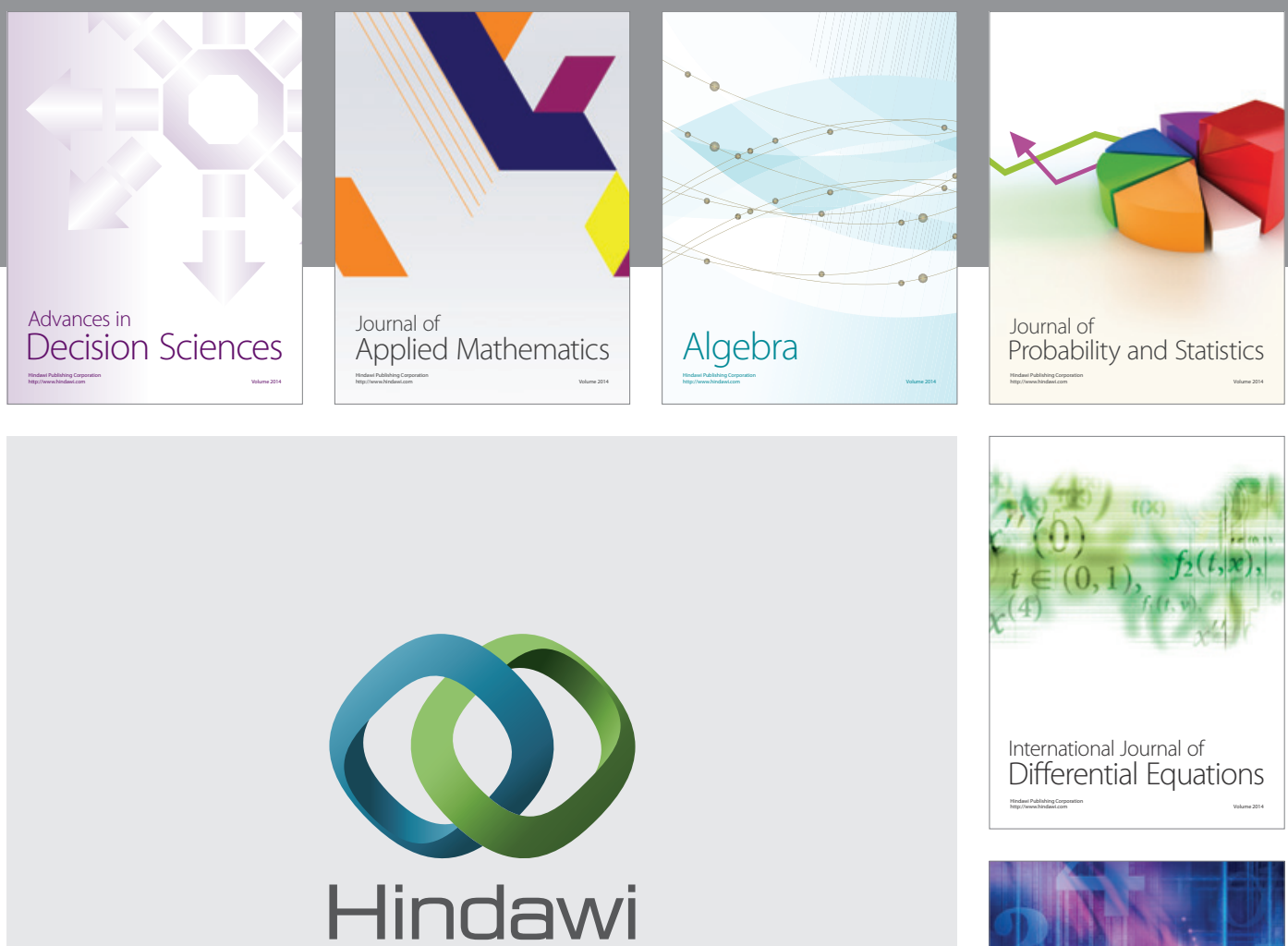

Submit your manuscripts at http://www.hindawi.com
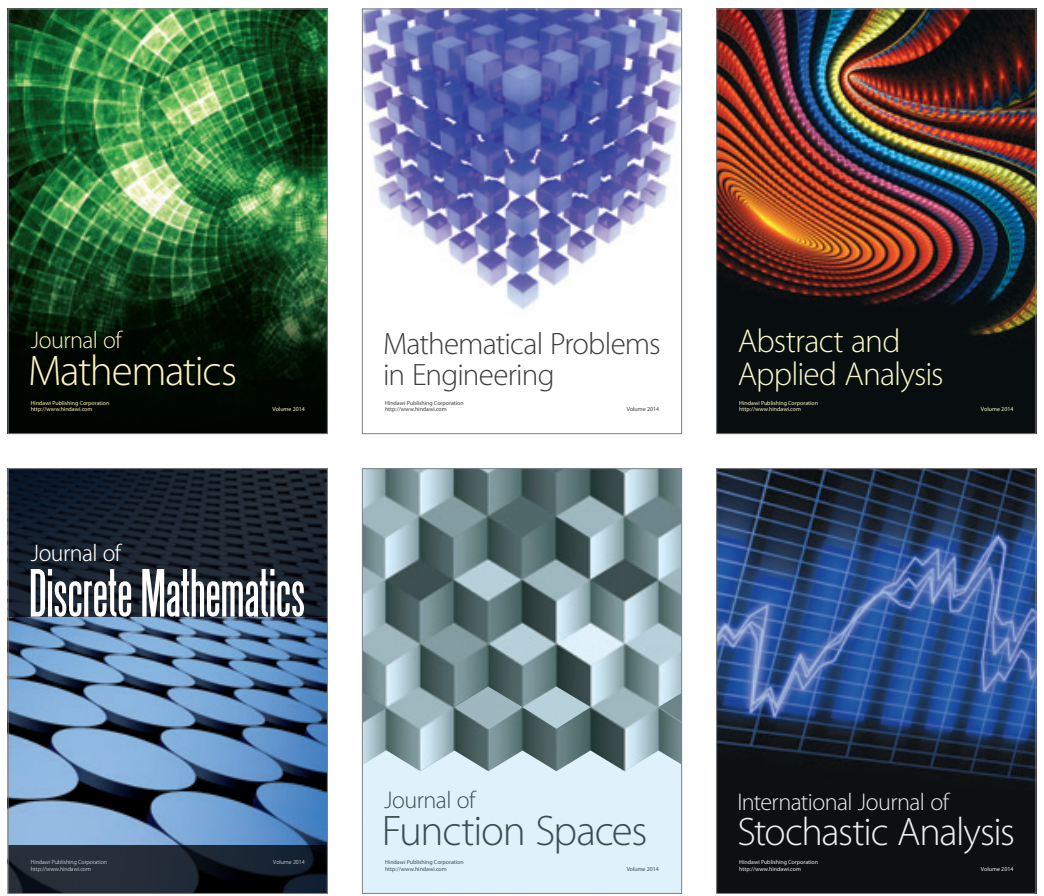

Journal of

Function Spaces

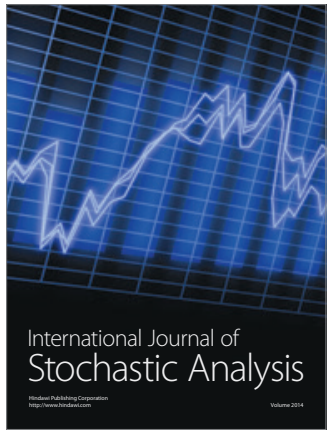

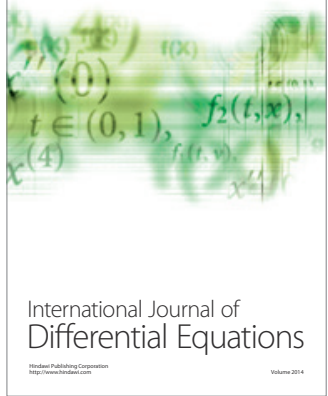
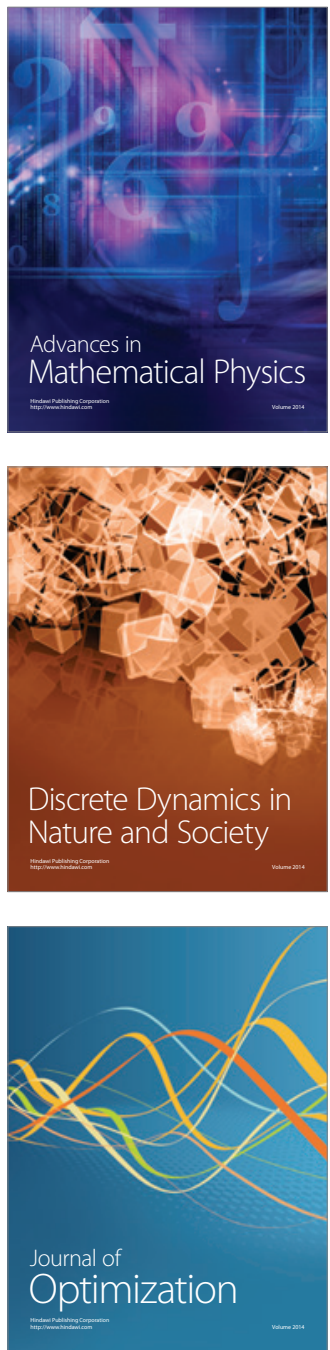